\title{
NEXT GENERATION OF FLEXIBLE ASSEMBLY SYSTEMS
}

\author{
Rolf Bernhardt, Dragoljub Surdilovic, Volker Katschinski, Gerhard Schreck and \\ Klaus Schröer
}

Rolf Bernhardt, Dragoljub Surdilovic, Volker Katschinski and Gerhard Schreck are with the Fraunhofer Institute for Production Systems and Design Technology (IPK), Pascalstraße 8-9, D-

10587 Berlin, Germany

(e-mail: \{rolf.bernhardt / dragoljub.surdilovic / volker.katschinski\}@ipk.fraunhofer.de). Klaus Schröer is with Volkswagen AG, PP-F2, Planung-Karosseriebau, Brieffach 1619/4, D-38436

Wolfsburg, Germany.

(e-mail:klaus.schroeer@volkswagen.de).

\begin{abstract}
In September 2006 an integrated European project was launched entitled "Flexible Assembly Systems through Workplace-Sharing and Time-Sharing Human-Machine Cooperation (PISA)". PISA belongs to the research area "next generation of flexible assembly technology and processes". The general aim of the project is to develop intelligent assist systems (IAS) in order to support the human worker instead of replace him. Thus, flexibility should not be reached through fully automated assembly systems but should instead support the better integration of human workers.
\end{abstract}

\section{INTRODUCTION}

The growing number of product variants, smaller lot sizes, accelerated time to market and shorter product life cycles have led to increasing demands on assembly equipment and concepts. They must achieve a high degree of flexibility with respect to variants, low-cost adaptability of products and quick amortisation within a sustainable equipment concept. In order to master these challenges, innovative approaches and technologies are required (Butala et al., 2002). The performance of existing automation techniques is often insufficient. As a solution to this problem, hybrid, i.e. human-integrated, approaches are proposed. The idea is to combine human flexibility, intelligence and skills with the advantages of sophisticated technical systems. Such systems should help the human worker instead of replace him. Intelligent assist systems (IAS) offer a rational, advanced method for the assembly of complex products on demand and at significantly reduced cost (Surdilovic et al. 2003). Since today neither the technology nor the tools for planning and managing IAS are available, the aim of the project is their prototypical development, including demonstration based on use cases [3]. One breakthrough of this project will be to bridge the gap between manual and automated assembly by introducing novel IAS technology and providing planning and integration tools to make this new technology applicable. A second breakthrough will be the reconfigurability of assembly systems and the reusability of assembly equipment. On the one hand, this is related to a modular structure of assembly systems including standard hardware and software interfaces for assembly equipment. On the other hand, methods and tools are needed for reconfiguration planning, reprogramming, life cycle and equipment management and knowledge bases for 
assembly solutions. Each breakthrough will lead to an increase in production capacity and productivity, to reduce the cost of investment and rearrangement and to react more quickly to market demands.

\section{PISA OBJECTIVES}

A skilled and motivated workforce still provides the most capable and reliable resource for the flexible (or customised) assembly of complex products. Recent studies have demonstrated clearly that flexibility can be improved by combining the benefits of human capabilities with sophisticated automation equipment in so-called hybrid flexible automation systems, providing a rational advanced concept for producing high-tech products with growing complexity at significantly reduced cost (Hägele et al., 2002). However, a reliable technological basis for hybrid systems does not yet exist, and the performance of existing flexible automation techniques (e.g. industrial robots) is quite limited in their ability to cooperate with and assist the human worker in a shared and reconfigurable assembly space (Karlsson, 2002). The existing standards do not permit workspace-sharing and cooperation.

Therefore, the prime objective of PISA is to establish a new generation of modular flexible assembly methodology by developing concepts, formal methods, standards and safety frameworks, tools and underlining technologies to allow the integration and cooperation between human workers and highly flexible devices and equipment in a qualitatively new and efficient manner. The main idea is to break from traditional paradigms regarding flexibility, cost, accessibility and applicability of high-tech assembly solutions, as well as conventional human-machine interaction. The project development concerns the following next-generation flexible assembly equipment and planning tools:

- A new generation of passive collaborative robots (COBOTS) and intelligent assist devices. These combine the benefits of industrial robots with those of passive handling devices. They also provide low-cost, operator-friendly solutions for the assembly of complex and variable volume products.

- Modular assembly robots representing the next generation of sensor-based robotic systems. These integrate visual and compliance control feedbacks, reconfigurable control systems and sophisticated grasping and tooling devices. They are capable of time-sharing with human workers and provide an efficient solution for capacity flexibility when workforce availability is lower or product volume varies.

- Assistant robots based on standard robotic systems. These are equipped with additional sensors and control functions and are capable of sharing a workspace and assembly process operations with a human worker. This approach offers a promising short-term solution for the flexible, reconfigurable assembly of highly customised products.

- Assembly process design and simulation tools involving the knowledge base of standardised processes and environmental models; interactive robots and human-in-loop models enabling a realistic conception of the assembly, planning and the optimisation of assembly system structures and process variations (virtual assembly system). These tools will also be very useful for the training and skill improvement of assembly workers.

- Reusable and reconfigurable electro-mechanical and control equipment; design and planning tools, including tight interfaces to assembly processes and parameters. This includes concepts and methods to support the planning and reconfiguration of newgeneration hybrid flexible assembly lines for specific production systems and market demands. 


\section{PISA STRUCTURE AND APPROACH}

Humans are the most flexible "components" of assembly systems, offering many advantages over machines (Tichem). Rather than remove human workers and develop fully automated solutions, PISA aims to keep human workers in the loop and to support them with qualified tools. Such an integrative approach combines human creativity, intelligence, knowledge, flexibility and skill with the advantages of sophisticated technical systems and tools, such as electronic and physical power, speed and accuracy.

The project is divided into nine interrelated subprojects (SP), as outlined in figure 1. The technical subprojects are SP1 to SP5; SP6 to SP8 handle innovation-related activities - IPR \& Exploitation, Training \& Dissemination and Occupational Safety \& Standards and SP9 comprises the project management.

The technical objective is to implement prototypical systems for the subprojects SP1 to SP4. Based on the use cases defined in SP5, integrated prototypes will be implemented to demonstrate the functionality, performance and novelty of the overall approach. Use cases will be selected from three different branches: the automotive, the home appliance and the aircraft industries. All prototypes will also be used for training purposes. Additional applications which are specifically interesting for SME will also be considered. This demonstrates one further advantage of the planned human-integrated solutions: not only can they be easily integrated into large assembly lines in the automotive and home appliance industries, but also into batch production in the aircraft industry.

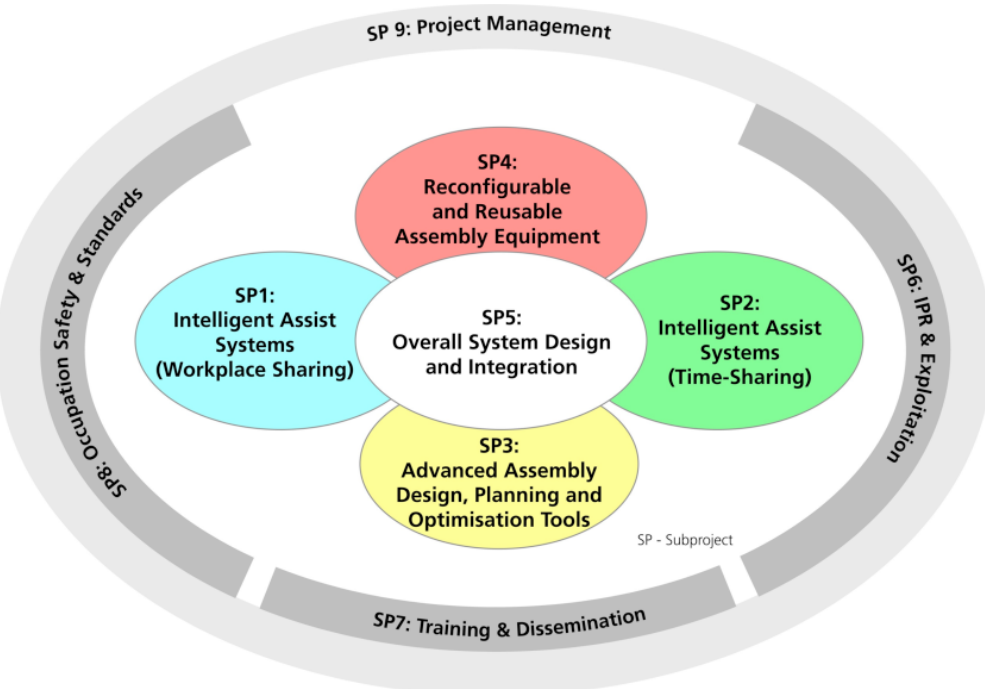

Figure 1. PISA Structure

\section{PISA TECHNICAL SUBPROJECTS}

SP1 Intelligent Assist Systems concerns two novel IAS for assembly processes. One is based on new industrial robot technology capable of sharing a workplace and operations with a human worker as outlined in figure 2 (ROBOT-IAS). The other, referred to as collaborating robot (COBOT), shown in figure 3, is capable of working jointly in direct physical contact with the human operator. 


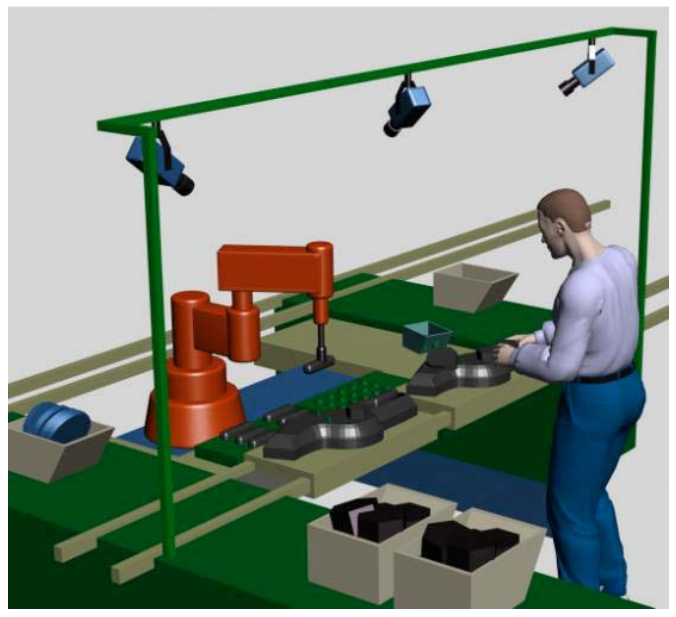

Figure 2. IAS Workplace-Sharing

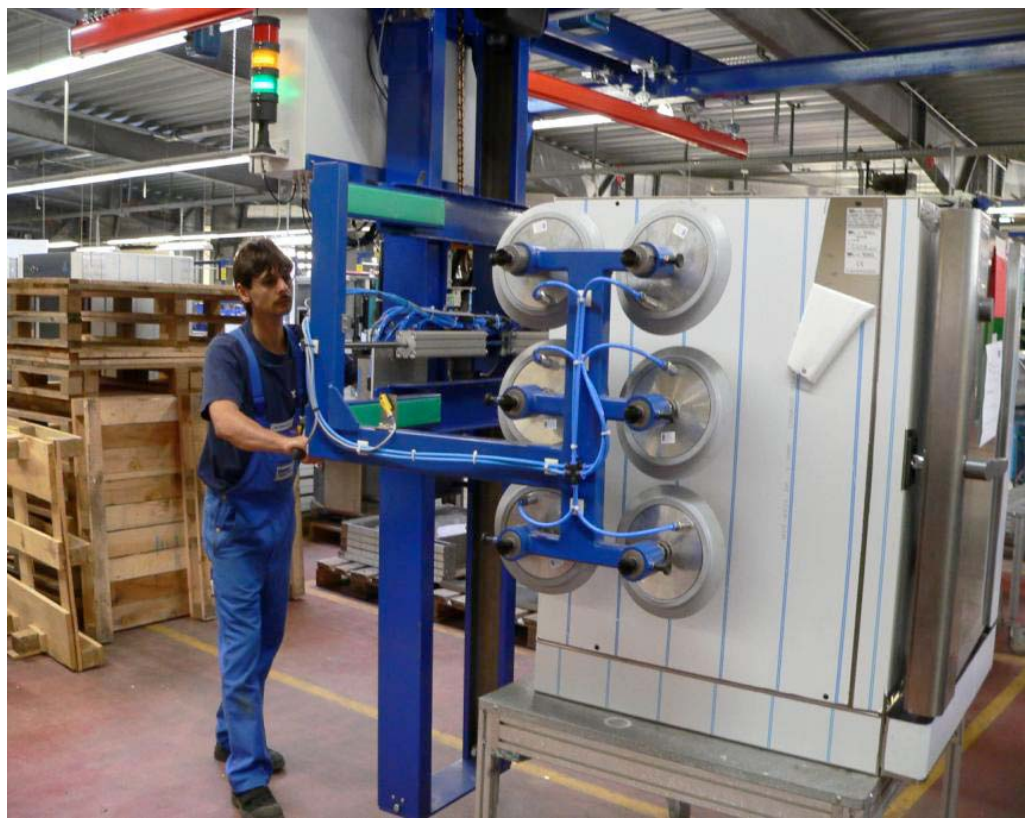

Figure 3. Cooperative Robot (COBOT)

Within SP1, control algorithms and prototypes are under development which will allow workplace-sharing as well as pure collaboration with human workers in assembly processes. This includes the design of a new intelligent COBOT prototype that integrates power assistance, motion guidance functions, advanced interaction control and programming algorithms into a reliable, efficient and ergonomic assembly system. Thereby it is important that sophisticated human-machine interfaces are developed which will ensure efficient cooperation and easy integration into assembly lines. A particular objective is to establish a technological and functional basis for safe operation (OSHA) and 
standardisation of the new assist systems. The advantages of the first European COBOT developed in SP1 in comparison to the pilot systems developed in the USA will be appreciably lower cost, significantly improved ergonomics, simpler intuitive operation, higher-precision rapid movements and considerably reduced stress during manipulation and assembly of complex and heavy loads.

The results of SP1 after one year are the user requirements for IAS. Based on a system analysis of actual needs within the considered branches (aerospace, automotive, household industry and SME assembly sectors), and on an analysis of the state-of-the-art and research technology, critical development needs and problems were identified and specified. These requirements provide the basis for the further development of the novel assist technology for human-centred assembly. Additionally, a first prototype was implemented, demonstrating some COBOT functions. This system was demonstrated at the $26^{\text {th }}$ international MOTEK fair in September 2007 in Stuttgart, Germany.

In SP2 Intelligent Assist Systems will be developed which will enable the timesharing of work between robots and humans (figure 4), depending on the lot size, required accuracy, complexity of assembly operation, etc. In order to apply these innovative human-machine systems efficiently, related planning tools will be developed.

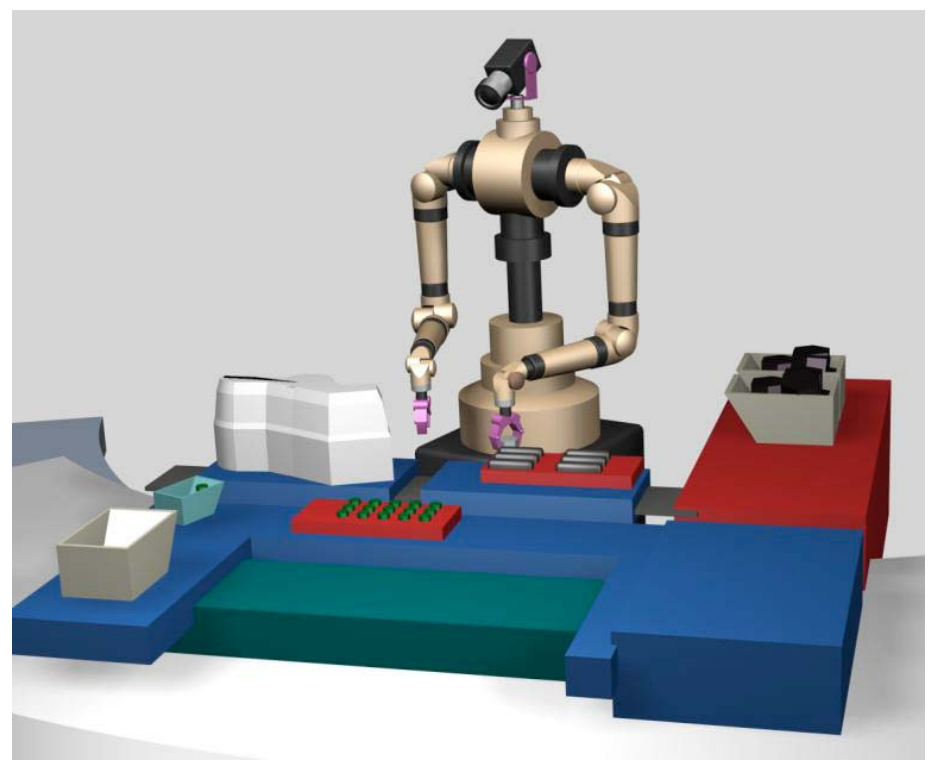

Figure 4. Time-Sharing Transportable Robot System

The specific objectives are the development of concepts, control algorithms and prototypes of intelligent assembly assist systems capable of time-sharing with human workers, i.e. replacement of humans when workforce availability is reduced or product volume changes during the product life cycle. This includes the design of a modular, easily programmable and transportable multi-arm robotic system which can work in an assembly workplace designed for human workers. The new assembly robot should represent the next generation of robotic systems integrating human dexterity and manipulation capabilities, visual and compliance control feedbacks, reconfigurable control systems and interfaces, as well as grasping and process-specific tooling devices. Additionally, a multi-modal human interface is in development which ensures efficient 
and skill-oriented programming by means of off-line programming and simulation tools, as well as virtual and augmented reality. Further aspects are related to occupational safety functions and systems as well as guidelines for the standardization of the new system.

The work began with the refinement of requirements (specifics from flexible assembly systems and end-user views) and the definition of the system architecture considering the assembly process and workplace environment. As a starting base system, existing anthropomorphic robots were considered. Based on the requirements, a system specification was worked out covering all subsystems such as mechatronic components, visual and force sensors, real-time controller, assembly task-level programming and safety functions. In the next step the software and hardware components will be designed. A simulation environment including kinematic and dynamic models will be developed and implemented in order to allow an analysis of component and overall system design. Based on these results the initial design will be revised and the components will be implemented.

The current results of SP2 are the user requirements on workplace-sharing robots capable of replacing the human operator in future assembly lines (referred to as an assembly robot). The concept of time-sharing robots for assembly processes is based on a dual-arm robot and focuses mainly on the improvement of capacity flexibility of advanced assembly. The main points are the overview of existing solutions and system requirements, the analysis and specification of the human-robot interface regarding the assembly process and safety issues and the specification of interfaces and requirements for simulation systems.

In SP3 Advanced Assembly Design Planning and Optimisation Tools are under development. An important feature of these tools will be their application in training purposes.

The specific objectives are to develop methods and tools based on concurrent engineering approaches which enable the use of virtual assembly for process design and system planning. Geometrical and non-geometrical features and knowledge of the product and assembly process must be integrated. The tools should also contain models of humanmachine collaboration for assembly system planning purposes.

Specifically, a toolset architecture based on open technologies and communication standards (figure 5) is in development. Thereby the novel collaborative human-machine approaches play a dominant role. The research work covers physical feature models of assembly process related components as well as tolerance and tolerance chain models. This includes the specification of a vendor-neutral structure and the development of a repository of these models. Regarding the human-robot cooperation, skill-oriented assembly planning tools will be developed, considering sequencing and scheduling based on manual and automatic assembly. Furthermore, an open knowledge base will be developed in order to ensure future access to assembly structures (product, process and system knowledge). Finally, a toolset prototype will be implemented, tested and evaluated using the IAS prototype environment.

The results of SP3 so far include the analysis of current technology used in planning and optimisation tools, as well as the definition of the technology development roadmap for SP3. This includes the input from end users gathered during the industrial workshops. The result summarises the state-of-art and describes the development necessary to meet the future demands in the area of assembly simulation, planning, scheduling and optimisation. Further work was done to introduce tools and methods to solve knowledge exchange challenges between the design, process planning, simulation and actual execution of manufacturing systems. 


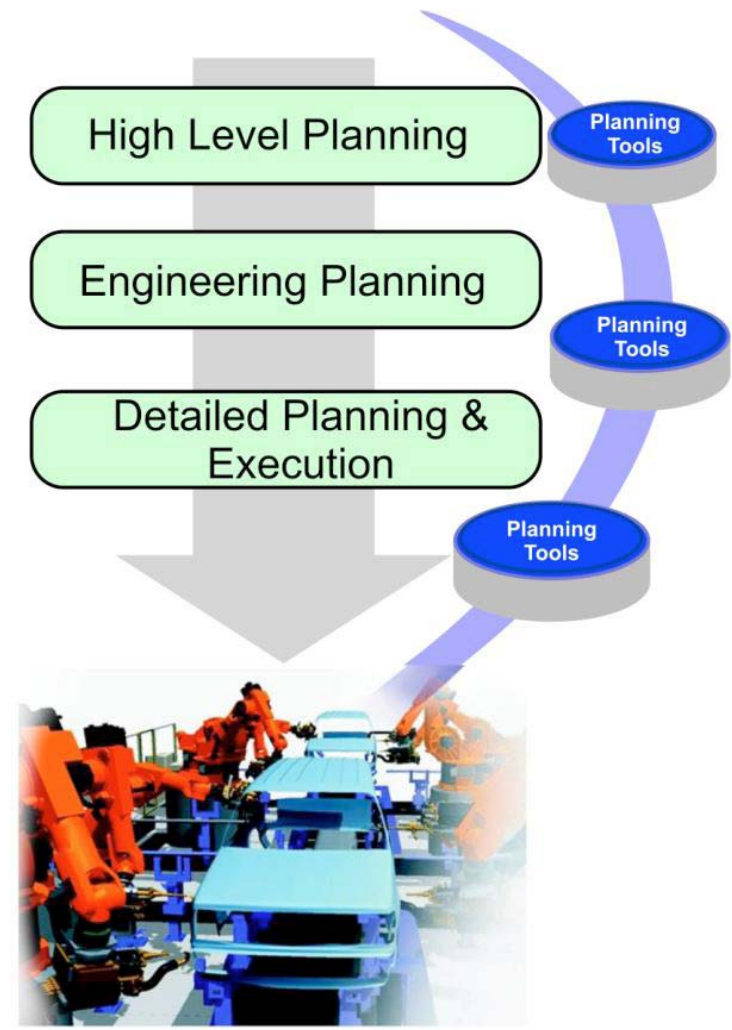

Figure 5. Assembly System Planning Tools

SP4 is dedicated to Reconfigurable and Reusable Assembly Equipment. Tools for the management of components, configuration, design, planning and control, as well as for simulation and testing will be developed.

The specific objectives are to develop reconfiguration concepts that support the rearrangement and reuse of assembly devices and systems considering generalised organisational, planning and technical requirements, as well as specific aspects of novel hybrid assembly technology. Thereby standardised hardware and software interfaces and tools enabling reconfigurability and reusability of assembly equipment will be considered. Based on the results, reconfigurable and reusable assembly system components will be designed, considering in particular the mechanical interfaces, condition monitoring systems and condition prediction algorithms. Finally, distributed control system hardware, software, and IT infrastructure for reconfiguration and reuse will be prototypically implemented.

The work began with a survey of end-user and system integrator companies in order to quantify the benefit/potential of reusability and reconfigurability of assembly equipment. Based on this survey an analysis of the strengths, weaknesses, opportunities and threats (SWOT-Analysis) was performed. The result is the requirements definition, which has been adapted to the specific needs of the industrial branches involved in PISA. In the next step a component knowledge base for life cycle assessment and reuse/reconfiguration management will be specified. This includes a formal description of tasks, equipment and life cycle information. A further important issue is related to the hardware of assembly 
system components, i.e. mechanical interfaces, condition monitoring and condition prediction algorithms. Related interface design rules as well as monitoring and prediction methods/tools will be developed and implemented. Additionally, assembly system controllers along with their hardware, software and IT infrastructure will be analysed. Based on this, a reusable/reconfigurable controller structure will be specified. Wherever applicable, these specifications are tested via simulation. The knowledge base and a distributed controller will be implemented.

In SP5 (Overall System Design and Integration), user requirements will be defined, the results of SP1-4 will be tested and evaluated and a demonstrator combining all results will be specified, implemented and evaluated.

The specific objectives are:

- to develop assembly system concepts that meet and balance the demands in terms of flexibility, cost-efficiency and technology level,

- to specify requirements on the performance and safety of new human-assisting assembly technology and on the planning methods and design tools supporting the novel technology and concepts,

- to assess prototype systems according to established demands,

- to integrate subproject prototypes and design overall demonstration systems, testing scenarios and evaluation criteria,

- to test the project demonstrators and examine the benefits considering reliability, flexibility, reusability and economical aspects and

- to define product design guidelines to optimally support new technology.

The work began with the consideration of bottlenecks in flexible assembly automation and defined research directions for the design of concepts for next-generation assembly systems. In figure 6 a COBOT prototype is shown which was specified and analysed for aircraft assembly.

The current results are the user requirements for reconfigurable and reusable assembly equipment. Based on a systematic analysis of standard hardware and software interfaces and control architectures, critical assembly problems were identified from the viewpoint of end-users and novel assembly technology developers, taking into account also the development of future assembly processes in European industry.

In particular, requirements have been specified for the further development of industrial robots to support power-assist applications in assembly processes. Furthermore, the deficiencies of existing assembly solutions in automotive-industry suppliers' assembly lines were analysed, and the requirements for new IAS for flexible assembly were specified, specifically from the point of view of system integration. 


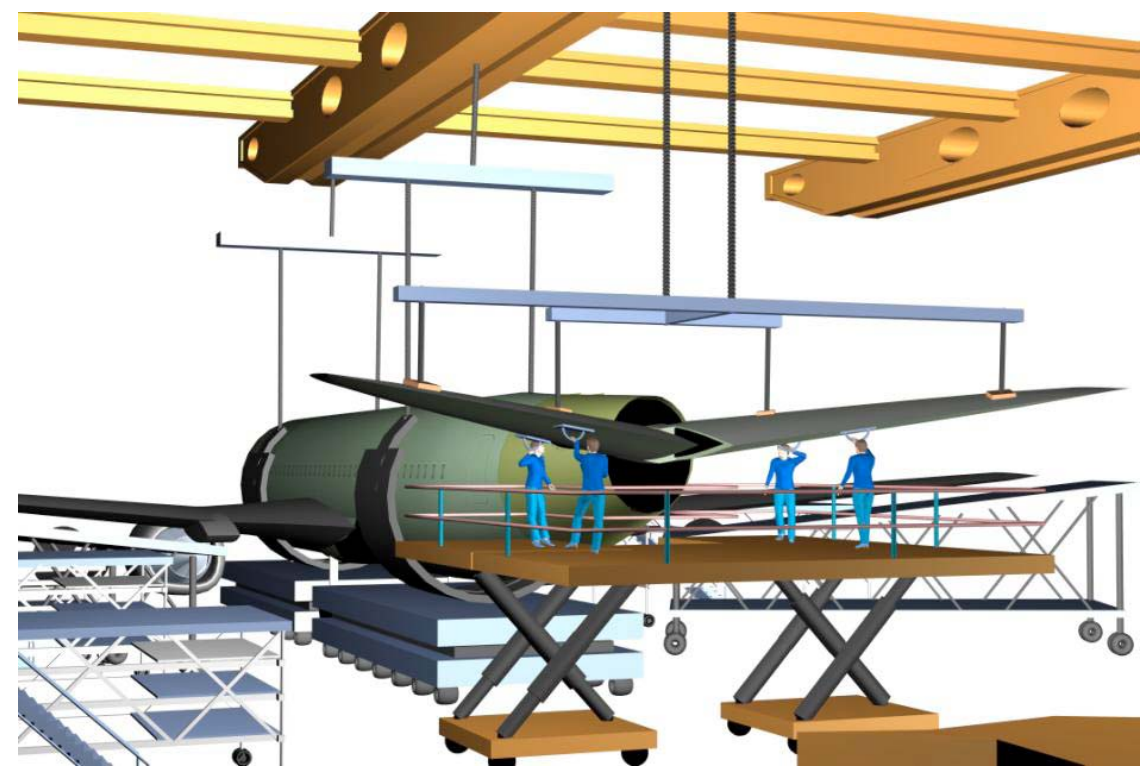

Figure 6. COBOT for Aircraft Assembly

\section{SUMMARY}

It is worth mentioning that the development of new concepts will be strongly supported by industrial research centres and SMEs. After the specification of requirements in the technology development subprojects, the work continues with the design of a detailed system architecture and the development of systems demonstration and testing scenarios. In order to reach the project goals, broad know-how in various scientific, engineering and technology areas from different branches is necessary. Partners selected for their practical and scientific perspectives provide this knowledge. The project involves four large companies, seven SME and seven research institutes from seven European countries. The integrated project approach enables parallel work on all subprojects and is therefore most efficient. The breakthrough of this IP will be the development of innovative intelligent assist systems (IAS) technology and the related methods and tools for planning, integration and reusability of assembly equipment. The results are flexible, cost-effective and highly productive solutions.

\section{CONCLUSION}

The IP-PISA targets an ambitious goal to develop a new generation of modular flexible assembly production concepts, formal methods, standards and safety framework tools and underlying technologies to allow the integration and cooperation between human workers and truly flexible, responsive devices in a qualitatively new and efficient manner. To reach this objective, the project is reasonably divided into four subprojects concerning assembly technology and tools development; one subproject dealing with the development and integration of assembly concepts and the evaluation of new technology; and three subprojects handling innovation-related activities. For SP1 to SP4 a duration of 36 months 
is planned. For SP5, which sets requirements for and integrates the results of SP1-SP4, and for the innovation-related subprojects, a duration of 48 months is planned.

\subsection{Acknowledgment}

The article reports on a research and technical development project partly funded by the EU as an Integrated Project in the $6^{\text {th }}$ Framework Programme.

\section{REFERENCES}

1. P. Butala, J. Kleine, S. Wingen, H. Gergs. “Assessment of assembly processes in European industry," in Proc. 35th CIRP-International Seminar on Manufacturing Systems, Seoul, May 2002.

2. D. Surdilovic, R. Bernhardt, and L. Zhang. New intelligent power-assist systems based on differential transmission. Robotica 2003; vol. 21: 295-302.

3. http://www.pisa-ip.org

4. M. Hägele, W. Schaaf, E. Helms, "Robot assistants at manual workplaces," in Proc. 33rd International Symposium on Robotics, 2002.

5. A. Karlsson. Assembly-initiated production - a strategy for mass-customisation utilising modular, hybrid automatic production systems. Assembly Automation 2002; vol. 22, no. 3: 239-247.

6. M. Tichem, "Position Report on Flexible Assembly Automation," Delft University: Laboratory for Production Engineering and Industrial Organisation, Delft University of Technology. Available: http://www.ocp.tudelft.nl/pto/research/publications/reports/KTHReport.pdf

7. OSHA: http://europe.osha.eu.int/ 